\title{
Transcriptomic Analysis of Human Endometrium Stromal Cells During Early Embryo Implantation (Within 48 Hour) in Vitro
}

\section{Shuo Han}

Beijing Chaoyang Hospital

Minghui Liu

Beijing Chao-Yang Hospital: Beijing Chaoyang Hospital

Shan Liu

Beijing Chao-Yang Hospital: Beijing Chaoyang Hospital

Yuan Li ( $\nabla$ cyliyuan@126.com )

Beijing Chaoyang Hospital

\section{Research}

Keywords: Embryo implantation, Endometrium, Pregnancy, Transcriptome

Posted Date: October 30th, 2020

DOI: https://doi.org/10.21203/rs.3.rs-99005/v1

License: (a) (i) This work is licensed under a Creative Commons Attribution 4.0 International License. Read Full License 


\section{Abstract}

\section{Background}

Successful embryo implantation is an essential prerequisite for pregnancy. Previous studies have shown that DNA methylation, histone, chromatin structure, and non-coding RNAs, microRNAs may participate in the regulation of gene expression during embryo implantation. However, the transcriptome changes of human endometrial stromal cells during early embryo implantation are not well characterized.

\section{Methods}

We cultured human endometrial stromal cells and simulated the process of embryo implantation in vitro. We further analyzed the endometrial transcriptome patterns of endometrial stromal cells in the preimplantation and post-implantation phase. We identified comprehensive transcriptomic profile of two endometrium stromal cells in particular developmental stage that may reflect the potential mechanism of embryo implantation.

Results

A total of 592 differentially expressed genes were identified after embryo implantation. Additionally, we identified key pathways (including TP53 and EGF signal pathway) that may regulate embryoendometrium interactions; our findings may serve as a foundation for targeted studies on endometrial receptivity and embryo implantation loss.

\section{Conclusion}

Our work showed the transcriptome changes of endometrial stromal cells within 48 hours after implantation which provides key insights into the crucial features of transcriptional regulation in the stepwise embryo development.

\section{Background}

Successful implantation of embryo is an essential prerequisite for pregnancy. This depends on a highly coordinated process between a viable embryo and receptive endometrium[1,2]. Recent years we have witnessed considerable advances in the development of in vitro embryo implantation model[3-6]. Postimplantation endometrial change in humans is a research hotspot; however, the implantation-related molecular mechanisms are not well characterized. Elucidation of the molecular mechanisms of implantation is a key imperative not only for the embryo development in vitro culture but also for the treatment of infertility and recurrent spontaneous abortion.

The process of implantation involves attachment and invasion of the uterine luminal epithelium. The blastocyst invades the uterine stroma and triggers several morphogenetic and signaling events. Microarray analyses have been performed to detect mRNAs of differentially expressed genes during 
embryonic implantation or the window of implantation in mice, rhesus monkey, and cattle[7][8][9]. These studies have identified several genes that are regulated during endometrial priming prior to embryonal implantation. However, there are considerable species-specific differences with respect to the postimplantation morphology in mammalian endometrium[10]. Therefore, characterization of the transcriptome changes of human endometrium during pre-implantation and post-implantation is a key imperative. However, owing to ethical constraints pertaining to human pregnancy, the transcriptome changes of human endometrial stromal cells (hESCs) during embryo implantation are not clear.

Successful establishment of in vitro embryo implantation model is critical. In this study, we cultured human hESCs in vitro and simulated the process of embryo implantation. We further analyzed the endometrial transcriptome patterns of hESCs during pre-implantation and post-implantation (48 hours). Additionally, we identified key pathways that may regulate embryo-endometrium interactions. Our findings may provide a foundation for targeted studies on endometrial receptivity and embryo implantation loss. Our work provides key insights into the crucial features of transcriptional regulation during embryo implantation.

\section{Materials And Methods}

\section{Culture of endometrial stromal cells}

Endometrial stromal cell were obtained from endometrial biopsy performed during the luteal phase. A small amount of endometrial tissue was placed in PBS solution of $10 \%$ fetal bovine serum, washed twice to remove blood cells and mucus, and cut into 1-2 mm pieces using eye scissors. After centrifugation, $0.25 \%$ trypsin plus $0.53 \mathrm{mM}$ EDTA solution was added for digestion at $37^{\circ} \mathrm{C}$ for 30 minutes; subsequently, the tissues were passed through a $100 \mu \mathrm{m}$ cell strainer (BD). The stromal cells pass through the cell strainer and were collected. After centrifugation and single wash, the cells were resuspended in $10 \% \mathrm{FBS}$ + DMEM/F12 medium and inoculated in a $35 \mathrm{~mm}$ dish. One hour later, the stromal cells had adhered to the wall.

\section{Embryo implantation experiment}

hESCs are primary cells within the first five generations. We used 96 well plates for embryo implantation experiment to reduce the number of stromal cells that were not in contact with the embryo. After 24-hour culture, the stromal cell culture medium was replaced with human embryo culture medium G2-plus (Vitrolife, Sweden), and the thawed-blastocyst was implanted on the stromal cell layer. The implantation performance was assessed 24 hours later. The adherent embryo was picked out 48 hours later and the stromal cells were collected for RNA sequencing.

This study was approved by the Ethics Review Board of Beijing Chao-Yang Hospital (2020-囚-279).

\section{Embryo frozen and thaw}


Spent poor-quality blastocysts were collected and frozen for research study. In the experiment group, 6 spent poor-quality blastocysts were from the same person. After thawing, the embryos were cultured in G2-plus (Vitrolife, Sweden) for 4 hours and then implanted in hESCs. The embryo freezing and thawing operation was performed as recommended in the KITAZATO reagent manual.

\section{RNA library construction and sequencing}

Trizol was used to extract total RNA. The NEBNext ${ }^{\circledR}$ Ultra ${ }^{\mathrm{TM}}$ II RNA Library Prep Kit for Illumina was used to construct the transcriptome library. The specific construction process is described online at www.international.neb.com.

\section{Quality assessment and quantification}

We used the Qubit Fluorometer to determine the library DNA concentration, which is qualified if it is greater than $1.0 \mathrm{ng} / \mathrm{L}$. The Qseq100 DNA analyzer was used to detect the library DNA length distribution. The criteria for qualification include concentration of length around 400bp, presence of single peaks, adapter less peaks, and absence of large fragment peaks. We used the KAPA Library Quantification Kit to quantify the molar concentration of library DNA as a standard for library mixing. After the library was mixed and denatured, it was added to the Illumina HiSeq sequencing platform for high-throughput sequencing. For specific operations, refer to the instruction manual. During sequencing, both ends of the library were sequenced separately (i.e., paired-end, PE); therefore, each sample generated two data files: reads1 (R1) and reads2 (R2).

\section{Data processing and analysis}

The RawData of high-throughput sequencing was filtered to obtain high-quality data (CleanData). Subsequently, the CleanData was compared with the designated reference genome (homo'u sapiens. grch38, annotation is enssemblhomo'u sapiens. Grch38.94) to calculate the efficiency of sequencing and reference genome, and to evaluate the saturation of sequencing data and gene coverage. The quality assessment report before and after data processing was generated by FastQC. Sequencing data quality control and processing were as follows: 1) The BWA algorithm was used to remove the low-quality regions at both ends of the sequence, using a mass threshold of 30. 2) Removal of joint sequence; 3 ) removal of 5 ' sequences containing fuzzy base $\mathrm{N} ; 4)$ removal of the sequences with length less than $60 \mathrm{bp}$ (usually 10bp).

\section{Construction of gene expression profile}

The gene expression profile was constructed to quantify gene expression and to calculate gene expression in different samples. The distribution of gene expression values in each sample are shown by box plot, density curve, and other illustrations. The known genes were identified including RNA, rRNA, tRNA, Premier RNA, Incrna, and novel genes.

\section{Differentially expressed testing}


Deseq normalization was performed among samples with different gene expression levels. Normalized Count was obtained from raw count and used to compare gene expression in different groups. Specific methods were followed to perform Audic ClaverieTest and Multiple Testing Correction: Benjamini Hochberg FDR; respective parameters: Maximum p value cut-off: 0.05 , fold-change cut-off: 2.0 .

\section{Functional enrichment analysis}

The differentially expressed genes were screened in different sample groups and subjected to cluster analysis; volcano maps were generated for visualization. GO/KEGG functional annotation and functional enrichment analysis of differential genes were performed to explore the functional and regulatory relationship of differentially expressed genes. In all tests, completely known genes were appointed as the background and $\mathrm{P}$ values (i.e., EASE score) indicating significant overlap between various gene sets were calculated using Benjamini-corrected modified Fisher's exact test. Only GO/KEGG-pathway terms associated with $P$ values $<0.05$ were considered significant and listed.

\section{Results}

\section{Human endometrial stromal cells culture and co-culture of embryo}

We confirmed the attachment of human blastocysts to stromal cells; therefore, we chose the primary hESCs within the first five generations as the bottom layer of cells, and chose the spent blastocysts from the same patient to eliminate experimental error attributable to differences in genetic background (Figure $1 \mathrm{~A}$ ). With ethical approval, 2 samples were primary hESCs (phESCs) and 6 samples were co-cultured with blastocytes (score: 4DD, 5DD and 6DD) (ehESCs). After co-culture of the embryo and stromal cells for 2 days, we found that all the blastocysts had attached to hESCs; $3 / 6$ blastocysts showed cell migration and invasion (Figure 1B).

\section{Global transcriptome profiling of human endometrial cells before and after embryo implantation}

After obtaining ethical approval, we performed RNA sequencing (RNA-seq) and analyzed the RNA-Seq data from 8 samples. After analysis, the transcriptional pattern of one ehESCs sample was similar to phESCs; therefore, we did not include it in the subsequent analysis. Principal component analysis demonstrated that the clustering of each population was segregated from each other (Figure 2A). In total, 592 genes were identified as DEGs according to similar criteria except for |log2FoldChangel > 1.5; these included 186 up-regulated and 406 down-regulated genes (Figure 2B).

Volcano plots showing the significance of the differential gene expression vs fold change are presented in Figure 3A. Figure 3B displays the top 15 upregulated and downregulated DEGs. PTGER1 and MMP16 have been reported in endometrial carcinoma research. The noted genes were related with oncology and decidualization (Figure 3B).

\section{Functional analysis of mRNA in hESCs}


The results of Gene Ontology (GO) annotation analyses indicated enrichment of DEGs in the following biological processes: (i) regulation of ribonucleoside monophosphate metabolic process; (ii) energy derivation by oxidation of organic compounds; (iii) protein targeting. DEGs were enriched in molecular function of NADH dehydrogenase activity and mitochondrial protein complex (Figure 4A-C).

We performed analysis on the basis of the Kyoto Encyclopedia of Genes and Genomes (KEGG) pathway database to identify the signal transduction pathways in these two comparisons; the results indicated that the genes were mainly correlated with oxidative phosphorylation, thermogenesis, ribosome and retrograde endocannabinoid signaling. TP53 and EGF signal pathway differed significantly (Figure 4D-E).

\section{Protein-protein interaction (PPI) network}

Network-based analysis was performed to understand the functions of predictive genes in terms of a PPI network topology. The STRING network was used for this purpose. The results showed 8 main networks of PPIs (Figure 5A). PPI network analysis was performed to identify the top 10 hub-gene targets. NADH ubiquinone oxidoreductase subunit related genes were found to play an important role in embryo implantation process (Figure 5B). COX6C and COX4I1 are the terminal enzymes of the mitochondrial respiratory chain which catalyze the electron transfer from reduced cytochrome $\mathrm{c}$ to oxygen.

\section{Discussion}

We performed a preliminary study of the transcriptome changes of hESCs in the early stage of human embryo implantation (within 48 hours). We identified significant changes in the transcriptome of hESCs after embryo implantation. 592 differentially expressed genes in the hESCs changed after implantation. Among these, metabolic gene expression changes involved the NADH/NAD+ redox cycle, tricarboxylic acid cycle, and electron transfer. TP53 signal pathway and EGF signal pathway activity were also changed. These findings indicate that hESCs undergo changes in the biological process of mitochondrial respiratory chain in order to provide a receptive environment for embryonal development postimplantation.

Among the differentially expressed genes, changes in the NADH ubiquinone oxidoreductase subunit related genes including NDUFA11 and NDUFB7 were the most striking. Their expression in the endometrium decreased significantly after embryo implantation within 48 hours. Mitochondrial respiratory complex I is composed of 45 different NADH ubiquinone oxidoreductase subunits. It transfers electrons from NADH to the respiratory chain[11].

The oxidative respiratory chain is related to the function of mitochondria. In previous studies, glucose metabolism level was found to decrease in the early stage (Day0-Day3) of embryonic development; in addition, there was inhibition of glycolysis and pentose phosphate pathways in mitochondria[12]. Before embryo implantation, NADPH participates in the decidualization of endometrium through the c-AMP pathway[13]; in addition, the mitochondrial function of stromal cells is active before implantation. Based 
on our results, we speculate that mitochondrial oxidative respiration of hESCs decreases to adapt to embryo development post-implantation.

Our results indicated downregulation of the TP53 signaling pathway of hESCs after implantation. In previous studies, the expression of TP53 protein was found to be increased in endometrial adenocarcinoma[14]. Moreover, the TP53 signal pathway was shown to be involved in decidualization of stromal cells[15]. The expression of TP53 protein in decidualized hESCs was increased in vitro. In the late secretory phase, the expression of TP53 increased[16]. Our study confirmed the suppression of the TP53 signal pathway activity of stromal cells after embryo implantation.

In our study, EGF signaling pathway was inhibited in hESCs after embryo implantation; however, EGF is indispensable for uterine growth[17]. Oncological studies have demonstrated abnormally high expression of EGFR in endometrial adenocarcinoma and upregulation of EGF signal pathway[18,19]. Heparin-binding epidermal growth factor (HP-EGF) regulates the decidualization of endometrium[20]. HP-EGF acts as a messenger in the endometrial-embryo interactions after embryo implantation. Inhibition of the EGF signaling pathway in hESCs may be related to the continued development of the embryo.

After 48 hours of embryo implantation, the main biological changes in hESCs include nucleotide synthesis and metabolism, mitochondrial protein synthesis, and ATP/NADPH energy metabolism. In a study of decidualization, hESCs were found to resist inflammation and oxidative damage[21]. Superoxide dismutase 2 (SOD2) in mitochondria helps hESCs migrate and promote the progression of endometriosis[22]. Thus, we speculate that the state of endometrial cells after implantation changes from the initial state of "attracting" embryo implantation to the state of "service" for embryos post-implantation; these changes provide a suitable environment for embryonic development.

Embryo implantation is a dynamic process. The more the in vitro model replicates the in vivo conditions, the more we can explore the internal mechanism of embryo implantation. There are two kinds of embryo implantation in vitro models, i.e., embryo contact model and embryo invasion model[2,23]. In this study, we used the embryo invasion model and by using next generation sequencing, we determined the transcriptome changes of hESCs pre/post-implantation.

Previous studies related to embryo implantation and endometrial receptivity were mainly focused on endometrial decidualization. A total of 161 human expression profile data were obtained using the keywords "hESCs" and "implantation" in GEO DataSets. Ethical constraints are a major hindrance to obtain tissue samples of human endometrium decidualization in the early stage after implantation; in vitro study of decidualization is mainly established by drug induction. The changes in endometrial transcriptome within 48 hours after embryo implantation have been studied only in animals. However, drug-induced models and animal experiments have their limitations and cannot really reflect the changes in human endometrium before and after implantation. This is the first study that employed the implantation model to study the endometrial transcriptome of human embryos after implantation (within 48 hours). Our study provides the human transcriptome information after embryo implantation and 
provides insights for further research on the internal mechanism of embryo implantation to improve the clinical implantation rate.

Our model attempted to recreate the process of embryo implantation in vivo and obtain the transcriptome closer to hESCs in vivo. Although we performed preliminary experiments to optimize the experiment condition, embryo implantation failure is liable to happen in vitro. In ART, there is a risk of failure of embryo transfer. In this study, embryo implantation failure may be related to embryo quality. We used abandoned embryos which have no clinical value; its effect on the changes of hESCs after embryo implantation require further research.

\section{Conclusion}

Our results showed the transcriptome changes of endometrial stromal cells within 48 hours after implantation which provides key insights into the crucial features of transcriptional regulation in the stepwise embryo implantation. This study provides a precise molecular description and definition for better preparation of endometrial for embryo implantation.

\section{Abbreviations}

hESCs, human Endometrial Stromal Cells

phESCs, primary human Endometrial Stromal Cells

ehESCs, human Endometrial Stromal Cells with embryo implantation

PPI, Protein-protein Interaction

KEGG, Kyoto Encyclopedia of Genes and Genomes

GO, Gene Ontology

EGF, Epidermal Growth Factor

HP-EGF, Heparin-binding Epidermal Growth Factor

\section{Declarations}

\section{Ethical Approval}

This study was approved by the Ethics Review Board of Beijing Chao-Yang Hospital (2020-邓-279).

\section{Consent for publication}

Not applicable. 
Availability of supporting data

Not applicable.

\section{Competing interests}

The authors declare no conflicts of interest

\section{Funding}

This study are funded by Beijing Health Promotion Foundation (2019-09-05) and Capital Health Development Scientific Research Project (Independent Innovation囚2020-1-2039),

\section{Acknowledgements}

We thanked all the family members who have support greatly for our science research career. Especially in China, young female physicians need control time precisely for the clinical work, housework and science research work.

\section{Authors' contributions}

S Han and MH Liu carried out the experiment. S Han and MH Liu wrote the manuscript with support from S Liu. S Liu helped supervise the project. Y Li. supervised the project. All authors read and approved the final manuscript.

\section{References}

[1] Blastocyst-endometrium interaction: Intertwining a cytokine network. Brazilian J Med Biol Res 2006;39:1373-85. https://doi.org/10.1590/S0100-879X2006001100001.

[2] In-vitro model systems for the study of human embryo-endometrium interactions. Reprod Biomed Online 2013;27:461-76. https://doi.org/10.1016/j.rbmo.2013.08.002.

[3] In-vitro model for stromal invasion during implantation of the human blastocyst | Human Reproduction | Oxford Academic n.d. https://academic.oup.com/humrep/article/18/2/283/639212 (accessed August 3, 2020).

[4] Experimental models for investigating implantation of the human embryo. Semin Reprod Med 2007;25:410-7. https://doi.org/10.1055/s-2007-991038.

[5] Models for study of human embryo implantation: choice of cell lines? Biol Reprod 2010;82:235-45. https://doi.org/10.1095/biolreprod.109.077800.

[6] Roadmap to embryo implantation: Clues from mouse models. Nat Rev Genet 2006;7:185-99. https://doi.org/10.1038/nrg1808. 
[7] Griffith OW, Chavan AR, Protopapas S, Maziarz J, Romero R, Wagner GP. Embryo implantation evolved from an ancestral inflammatory attachment reaction. Proc Natl Acad Sci U S A 2017;114:E6566-75. https://doi.org/10.1073/pnas.1701129114.

[8] Ma X, Li P, Zhang Q, He L, Su G, Huang Y, et al. Transcriptome analysis of the endometrium from Chinese Erhualian sows that differ in calcium ion concentration and litter size. Anim Genet 2019;50:32633. https://doi.org/10.1111/age.12788.

[9] Moraes JGN, Behura SK, Geary TW, Hansen PJ, Neibergs HL, Spencer TE. Uterine influences on conceptus development in fertility-classified animals. Proc Natl Acad Sci U S A 2018;115:E1749-58. https://doi.org/10.1073/pnas.1721191115.

[10] Bauersachs S, Ulbirch SE, Gross K, Schmidt SEM, Meyer HHD, Wenigerkind H, et al. Embryo-induced transcriptome changes in bovine endometrium reveal species-specific and common molecular markers of uterine receptivity. Reproduction 2006;132:319-31. https://doi.org/10.1530/rep.1.00996.

[11] Mitochondrial Respiratory Complex I: Structure, Function and Implication in Human Diseases. Curr Med Chem 2009;16:1266-77. https://doi.org/10.2174/092986709787846578.

[12] Glycogen and Glucose Metabolism Are Essential for Early Embryonic Development of the Red Flour Beetle Tribolium castaneum. PLoS One 2013;8. https://doi.org/10.1371/journal.pone.0065125.

[13] NADPH oxidase-derived reactive oxygen species mediate decidualization of human endometrial stromal cells in response to cyclic AMP signaling. Endocrinology 2011;152:730-40.

https://doi.org/10.1210/en.2010-0899.

[14] p53 overexpression in advanced-stage endometrial adenocarcinoma. Am J Obstet Gynecol 1996;175:1246-52. https://doi.org/10.1016/S0002-9378(96)70036-4.

[15] Interpretation of p53 immunohistochemistry in endometrial carcinomas: toward increased reproducibility. NcbiNImNihGov n.d.

[16] Wild-type p53 protein is up-regulated upon cyclic adenosine monophosphate-induced differentiation of human endometrial stromal cells. J Clin Endocrinol Metab 2004;89:5233-44.

https://doi.org/10.1210/jc.2004-0012.

[17] Uterine and vaginal organ growth requires epidermal growth factor receptor signaling from stroma. AcademicOupCom n.d.

[18] Endometrial stromal sarcomas frequently express epidermal growth factor receptor (EGFR, HER-1) n.d.

[19] Epidermal growth factor receptor signaling enhanced by long-term medroxyprogesterone acetate treatment in endometrial carcinoma. Elsevier n.d. 
[20] Heparin-binding epidermal growth factor and its receptors mediate decidualization and potentiate survival of human endometrial stromal cells. J Clin Endocrinol Metab 2005;90:913-9. https://doi.org/10.1210/jc.2004-0476.

[21] Heparin prevents oxidative stress-induced apoptosis in human decidualized endometrial stromal cells. Med Mol Morphol 2019;52:209-16. https://doi.org/10.1007/s00795-019-00220-x.

[22] Mitochondria and oxidative stress in ovarian endometriosis. Free Radic Biol Med 2019;136:22-34. https://doi.org/10.1016/j.freeradbiomed.2019.03.027.

[23] Experimental models for investigating implantation of the human embryo. Semin Reprod Med 2007;25:410-7. https://doi.org/10.1055/s-2007-991038.

\section{Figures}

A
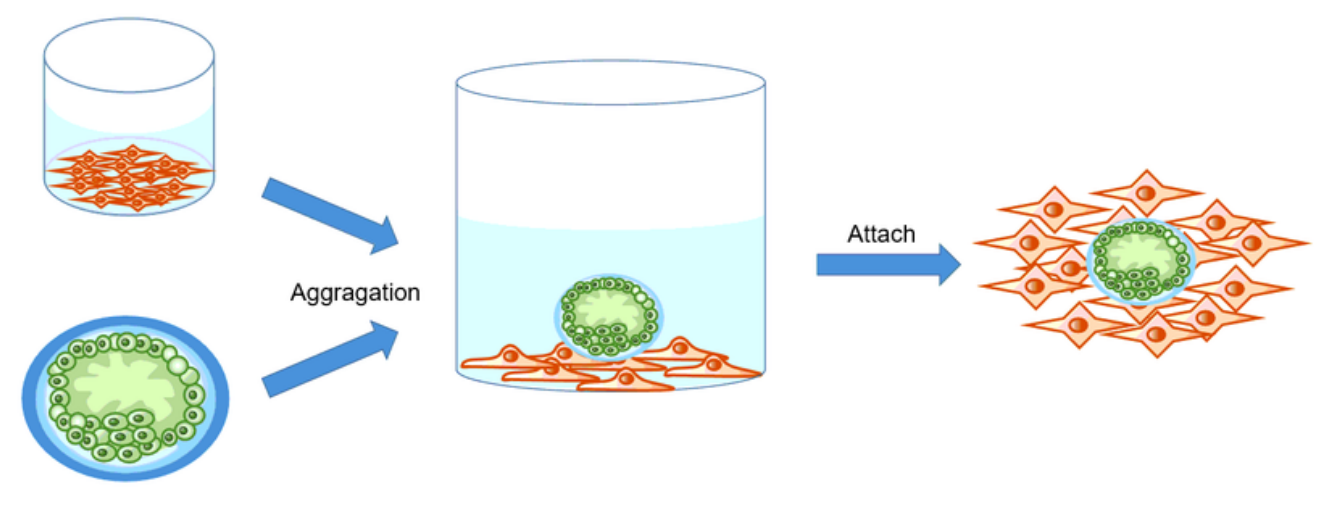

B
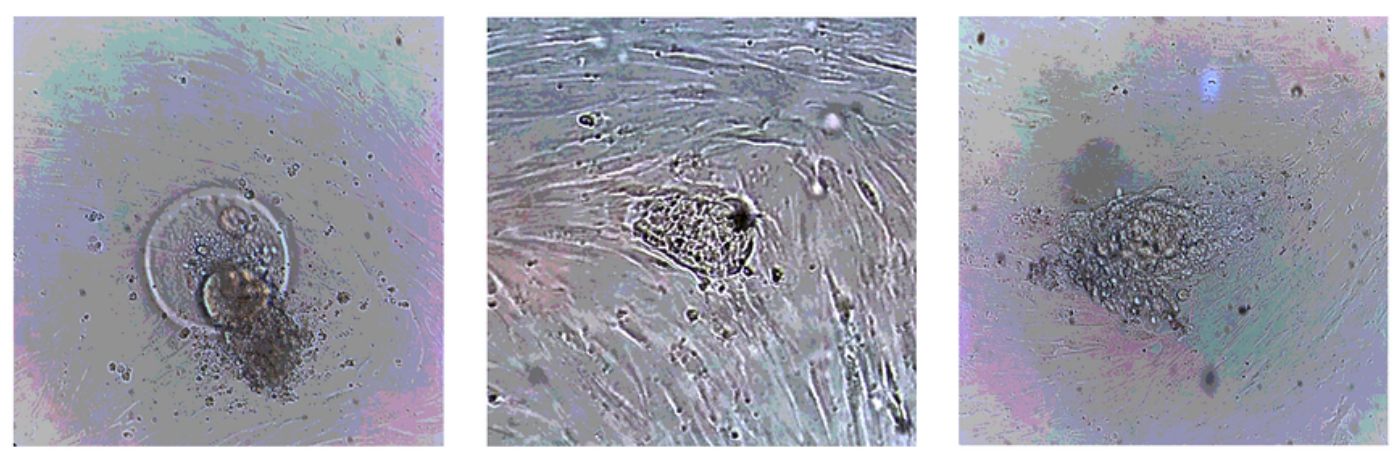

\section{Figure 1}

The process of embryo co culture with endometrial stromal cells (hESCs) and embryo implantation. A: The process of embryo implantation in hESCs was simulated in vitro. B: Embryo implantation in vitro was observed under inverted microscope. 


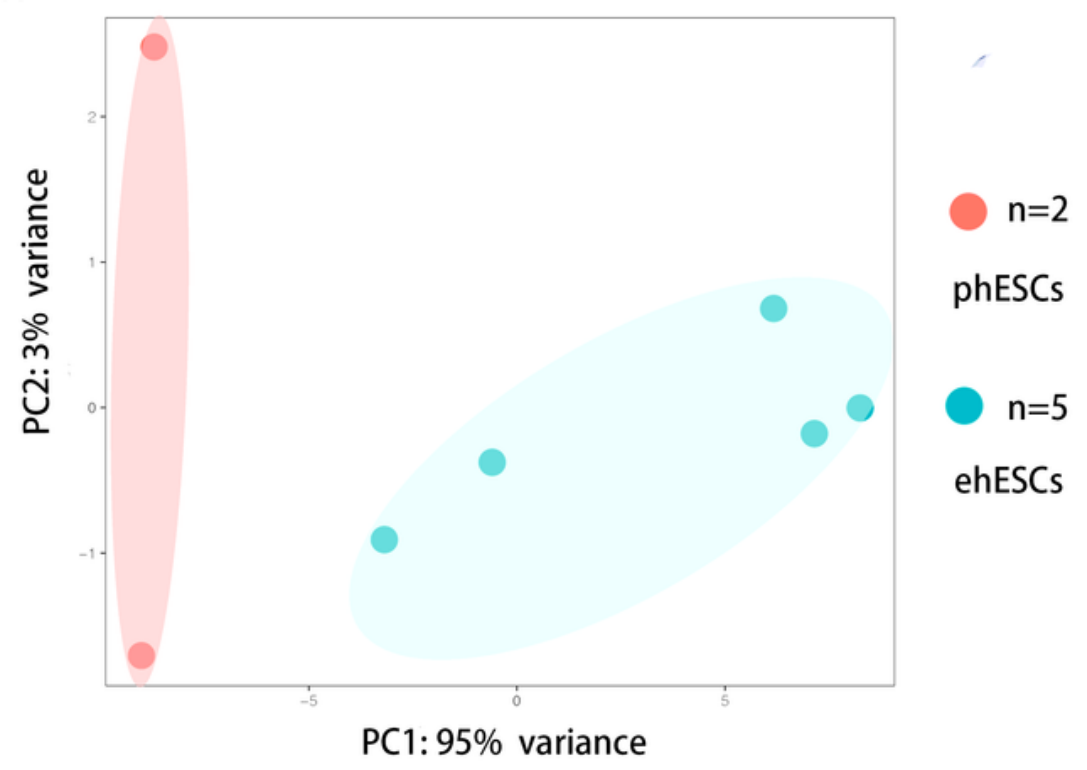

B

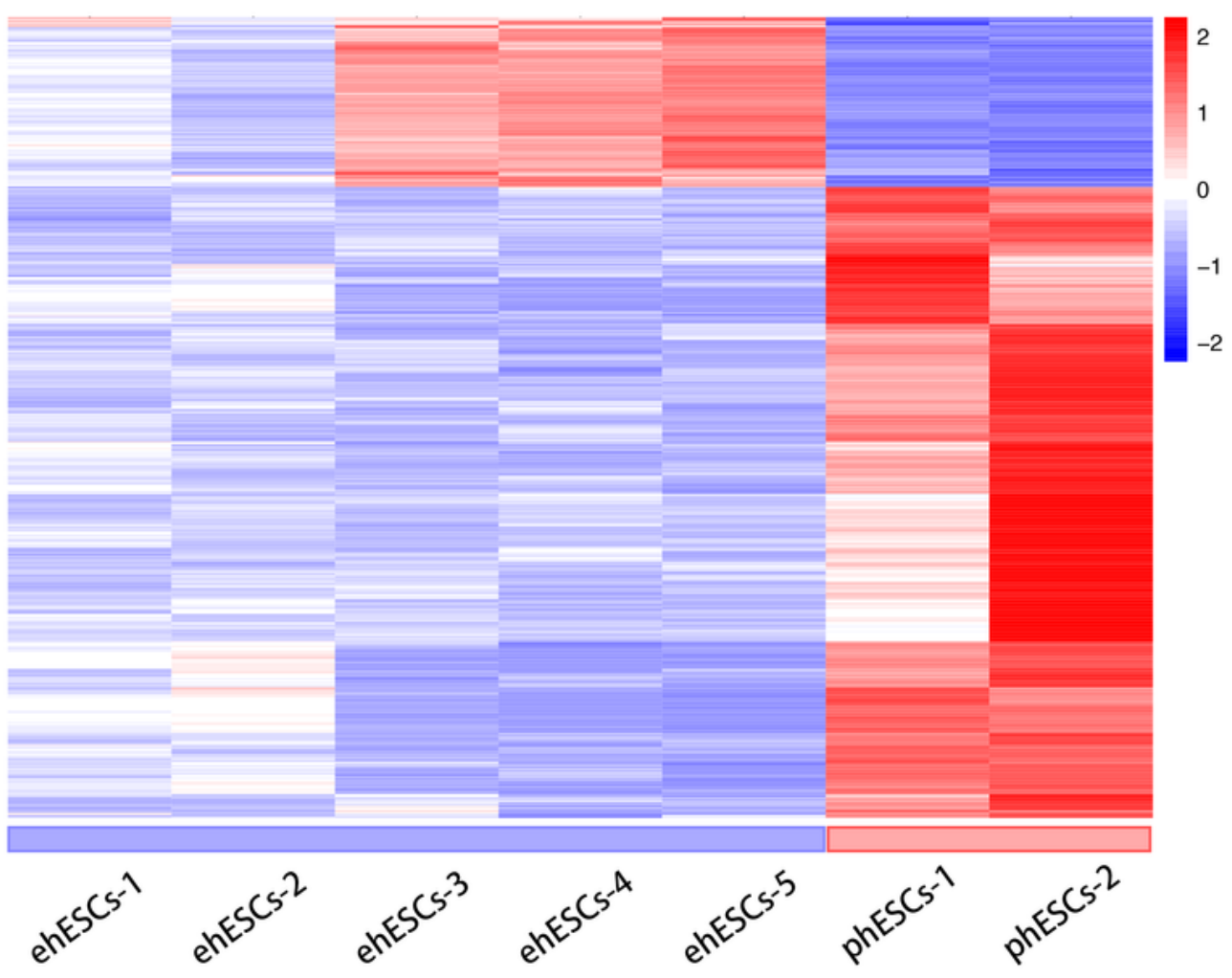

\section{Figure 2}

Transcriptome pattern of phESCs and ehESCs. A: Principal component analysis showed samples to cluster by cell population. B: Cluster analysis of differentially expressed genes among the severn samples. 


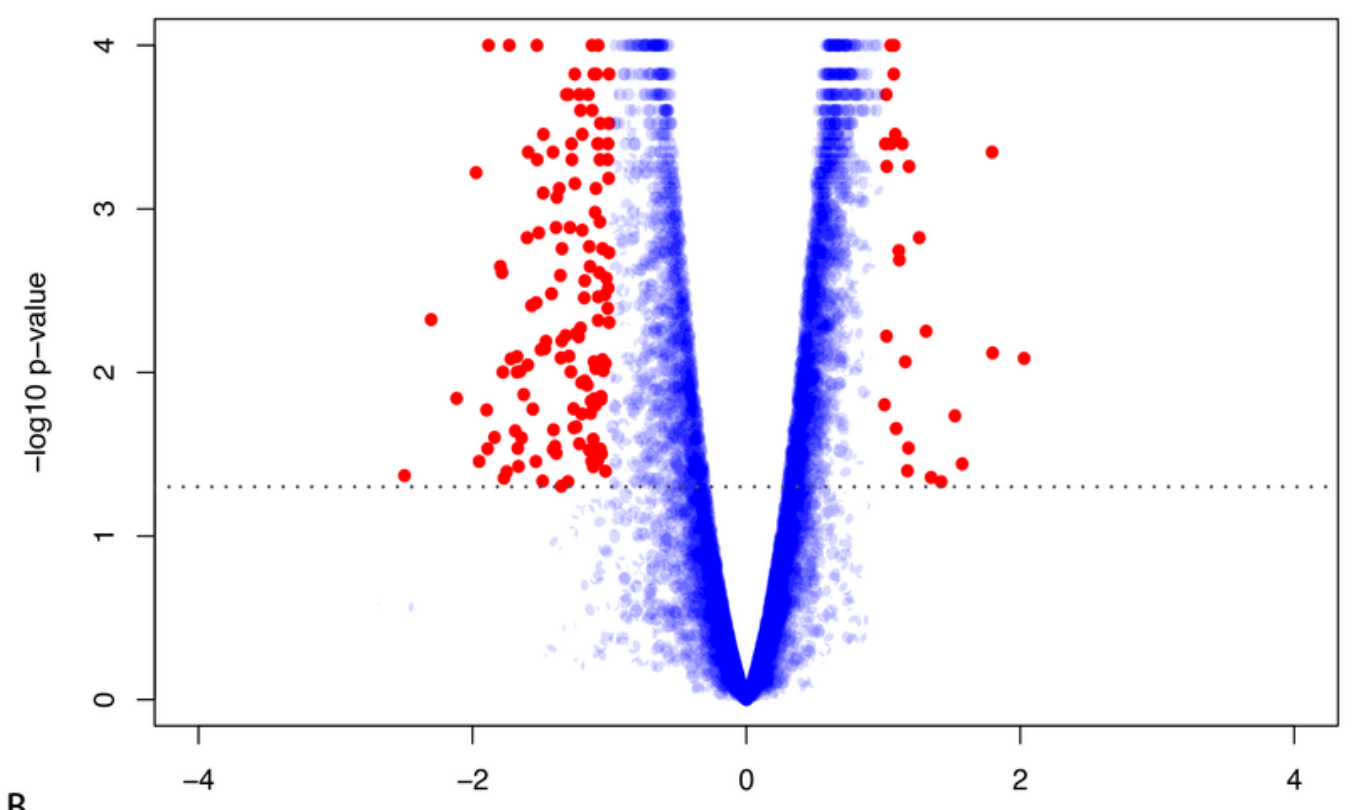

B

log2 Fold Change

\begin{tabular}{|c|c|c|c|c|c|}
\hline Gene_id & gene symble & Regulation & Name & Oncology & Decidualization \\
\hline XLOC_007184 & PCDH9 & up & Protocadherin 9 & $*$ & + \\
\hline XLOC_027564 & JADE3 & up & Jade Family PHD Finger 3 & * & \\
\hline XLOC_021696 & PHF3 & up & PHD Finger Protein 3 & & \\
\hline XLOC_026074 & MMP16 & up & Matrix Metallopeptidase 16 & ** & + \\
\hline XLOC_019243 & FGF5 & up & Fibroblast Growth Factor 5 & * & + \\
\hline XLOC_020023 & FAT1 & up & FAT Atypical Cadherin 1 & * & + \\
\hline XLOC_001012 & RASAL2 & up & RAS Protein Activator Like 2 & * & \\
\hline XLOC_021902 & UTRN & up & Utrophin & * & \\
\hline XLOC_026157 & SAMD12 & up & Sterile Alpha Motif Domain Containing 12 & & + \\
\hline XLOC_004469 & CBL & up & Cbl Proto-Oncogene & * & \\
\hline XLOC_026484 & VPS13A & up & Vacuolar Protein Sorting 13 Homolog A & * & \\
\hline XLOC_014156 & FOXN2 & up & Forkhead Box N2 & * & \\
\hline XLOC_025403 & $\mathrm{ESCO} 2$ & up & Inromatia conesion IN-Acetyitra & * & \\
\hline XLOC_006178 & KRAS & up & KRAS Proto-Oncogene, GTPase & * & + \\
\hline XLOC_001360 & HES4 & down & Hes Family BHLH Transcription Factor 4 & * & \\
\hline XLOC_020454 & PCDHA1 & down & Protocadherin Alpha 1 & $*$ & \\
\hline XLOC_018974 & C4orf48 & down & Chromosome 4 Open Reading Frame 48 & & \\
\hline XLOC_009206 & ANTKMT & down & Adenine Nucleotide Translocase Lysine Methyltransferase & & \\
\hline XLOC_013393 & PTGER1 & down & Prostaglandin E Receptor 1 & $* *$ & + \\
\hline XLOC_014829 & RAMP1 & down & Receptor Activity Modifying Protein 1 & & + \\
\hline XLOC_013542 & COX7A1 & down & Cytochrome C Oxidase Subunit 7A1 & * & \\
\hline XLOC_010976 & NDUFAF8 & down & NADH:Ubiquinone Oxidoreductase Complex Assembly Factor 8 & & \\
\hline XLOC_000011 & ISG15 & down & ISG15 Ubiquitin Like Modifier & * & + \\
\hline XLOC_001659 & EVA1B & down & Eva-1 Homolog B & & \\
\hline XLOC_012565 & NDUFA13 & down & NADH:Ubiquinone Oxidoreductase Subunit A13 & * & \\
\hline XLOC_013955 & A1BG & down & Alpha-1-B Glycoprotein & * & \\
\hline XLOC_012379 & TGFBR3L & down & Transforming Growth Factor Beta Receptor 3 Like & * & \\
\hline XLOC_009240 & NPW & down & Neuropeptide W & & \\
\hline XLOC_012808 & APOE & down & Apolipoprotein E & & + \\
\hline
\end{tabular}

\section{Figure 3}

Differential expression genes (DEGs) of pre-/post-implantation A: Volcano plot highlight significant genes. B: Top15 down and up regulated genes are listed in the table. 
A

C
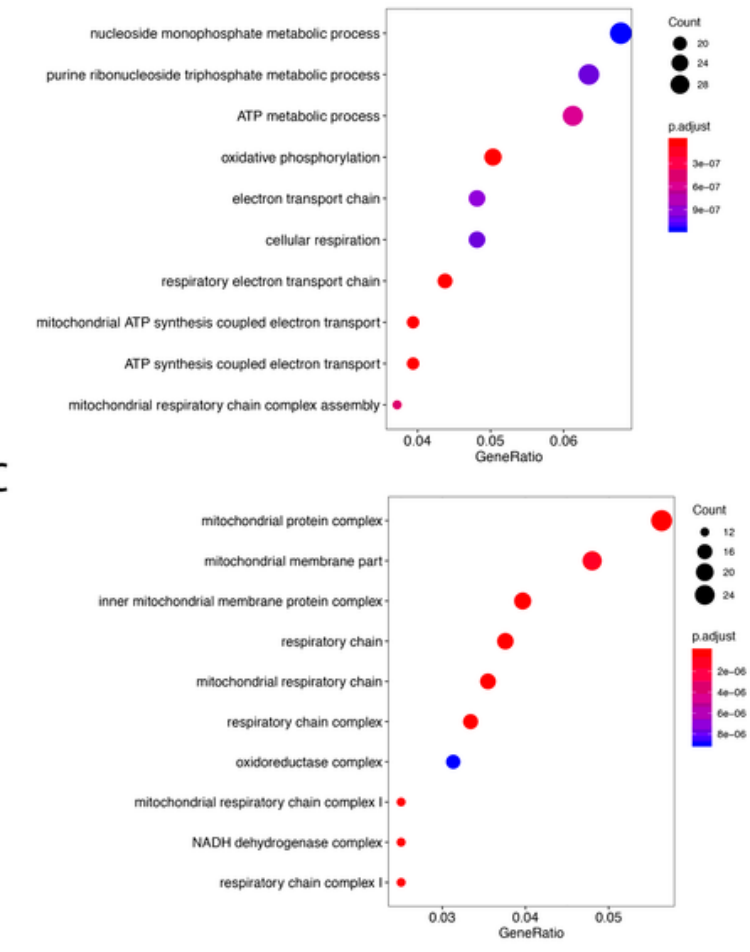

D

$E$

\begin{tabular}{lcl}
\hline \multicolumn{1}{c}{ Pathway } & Gene regulate number & \multicolumn{1}{c}{ Genes } \\
\hline p53 pathway feedback loops 2 & $4 / 46$ & HRAS, RB1, MAPK12, KRAS \\
EGF receptor signaling pathway & $5 / 114$ & HRAS, RASAL2, MAPK12, CBL, NF1 \\
Cholesterol biosynthesis & $2 / 12$ & PMVK, MVD \\
TGF-beta signaling pathway & $4 / 91$ & HRAS, EP300, JUN, DMAPK12 \\
\hline
\end{tabular}

\section{Figure 4}

Gene functional analysis of phESCs and ehESCs (GO and KEGG analysis). A-C囚Bubble plot of representative $\mathrm{GO}$ enrichment results of differentially expressed genes. DQThe Bubble Chart showed the top 9 of mRNAs KEGG pathway enrichment results. E: The top 4 PNATHER pathway. 
A

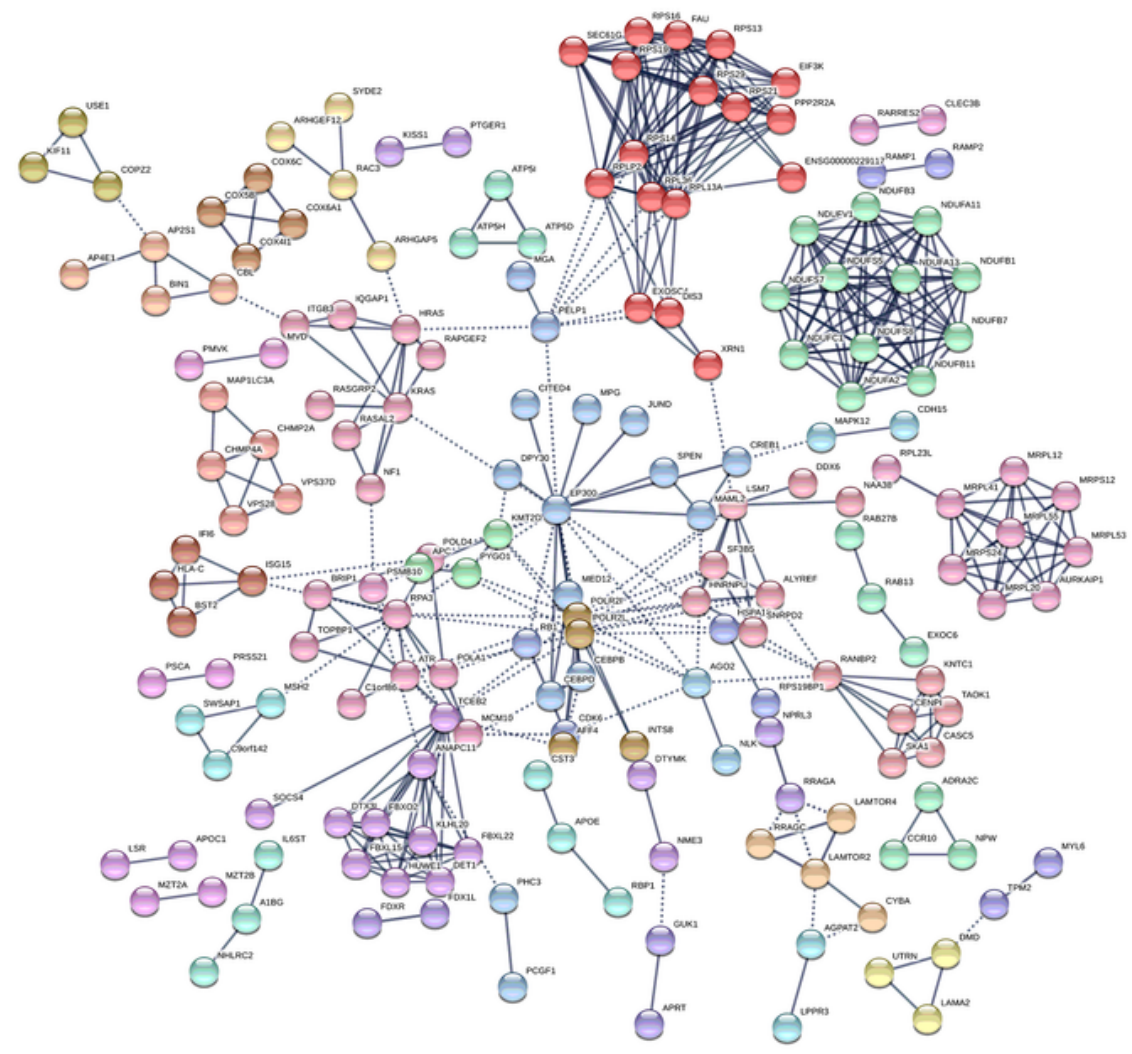

B

\section{C}

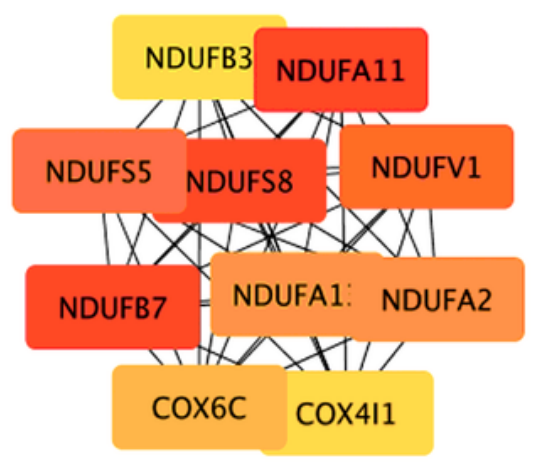

\begin{tabular}{cll}
\multicolumn{3}{c}{ Top 10 hubgene in network by MCC method } \\
\hline Rank & Rank & Score up/down \\
\hline 1 & NDUFA11 & $1.25 \mathrm{E}+10$ DOWN \\
2 & NDUFB7 & $1.25 \mathrm{E}+10$ DOWN \\
3 & NDUFS8 & $1.25 \mathrm{E}+10$ DOWN \\
4 & NDUFV1 & $1.25 \mathrm{E}+10$ DOWN \\
5 & NDUFS5 & $1.25 \mathrm{E}+10$ DOWN \\
6 & NDUFA2 & $1.25 \mathrm{E}+10$ DOWN \\
7 & NDUFA13 & $1.25 \mathrm{E}+10$ DOWN \\
8 & COX6C & $1.25 \mathrm{E}+10$ DOWN \\
9 & COX411 & $1.25 \mathrm{E}+10$ DOWN \\
10 & NDUFB3 & $1.25 \mathrm{E}+10$ DOWN \\
\hline
\end{tabular}

\section{Figure 5}

Protein-protein interaction analysis. A: The figure illustrates the PPI networks of the DEGs based on the STRING database. B-C: PPI network of 10 hubgenes extracted from (A). 